\title{
Three-dimensional modulated spin liquid model applied to $\mathrm{URu}_{2} \mathrm{Si}_{2}$
}

\author{
Christopher Thomas, ${ }^{1}$ Sébastien Burdin, ${ }^{2,3}$ Catherine Pépin, ${ }^{4}$ and Alvaro Ferraz ${ }^{1,5}$ \\ ${ }^{1}$ International Institute of Physics, Universidade Federal do Rio Grande do Norte, 59078-400 Natal-RN, Brazil \\ ${ }^{2}$ Université de Bordeaux, LOMA, UMR 5798, F-33400 Talence, France \\ ${ }^{3}$ CNRS, LOMA, UMR 5798, F-33400 Talence, France \\ ${ }^{4}$ Institut de Physique Théorique, CEA-Saclay, F-91191 Gif-sur-Yvette, France \\ ${ }^{5}$ Departamento de Física Teórica e Experimental, Universidade Federal do Rio Grande do Norte, 59072-970 Natal-RN, Brazil
}

(Received 29 June 2012; revised manuscript received 20 November 2012; published 18 January 2013)

\begin{abstract}
We have developed a three-dimensional version for the modulated spin liquid in a body-centered tetragonal lattice structure to describe the hidden order observed in $\mathrm{URu}_{2} \mathrm{Si}_{2}$ at $T_{0} \approx 17.5 \mathrm{~K}$. This second-order transition is well described by our model and confirms our earlier hypothesis. The symmetry of the modulation is minimized for $\mathbf{Q} \equiv(1,1,1)$. We assume a linear variation of the interaction parameters with the lattice spacing and our results show good agreement with uniaxial and pressure experiments.
\end{abstract}

DOI: 10.1103/PhysRevB.87.014422

PACS number(s): 75.10.Kt, 72.80.Ga, 74.40.Kb, 75.25.Dk

The fascinating hidden-order phase observed in the heavy fermion $\mathrm{URu}_{2} \mathrm{Si}_{2}$ below $T_{0}=17.5 \mathrm{~K}$ is the center of great discussion concerning the origin of the mechanism for this second-order transition. ${ }^{1,2}$ From thermodynamics properties, a huge entropy quench is observed and this cannot be explained by a conventional antiferromagnetic transition due to the small value of the magnetic moment in this phase.,

The phase diagram obtained for the $\mathrm{URu}_{2} \mathrm{Si}_{2}$ is very interesting. ${ }^{5-8}$ In ambient pressure, the system undergoes a second-order transition at $T_{0}=17.5 \mathrm{~K}$ to a phase known as hidden order $(\mathrm{HO})$ with a small magnetic moment $\mu \approx$ $0.02 \mu_{B}$. At very small temperature, a superconducting phase is found below $T \approx 1.5 \mathrm{~K}$. Applying hydrostatic pressure or uniaxial stress $\left(\sigma_{x}^{a}\right)$, the system turns into an antiferromagnetic (AF) phase with a magnetic moment $\mu \approx 0.4 \mu_{B}$ for $P_{x} \approx$ $0.5 \mathrm{GPa}$ or $\sigma_{x}^{a} \approx 0.33 \mathrm{GPa}$, respectively. Bakker et al. ${ }^{9}$ showed that $T_{0}$ increases linearly with the uniaxial stress applied along the $a$ axis and decreases when the uniaxial stress is applied along the $c$ axis. Elastic neutron-scattering measurements with uniaxial stress applied along $[1,0,0],[1,1,0]$, and $[0,0,1]$ directions show a very sensitive variation of the ordered moment. ${ }^{10}$ In-plane stress increases $\mu$, while a perpendicular stress does not change the ordered moment at all. The tuning parameter to the HO-AF transition seems to be the in-plane lattice constant $a$, as shown by Bourdarot et $a l^{8}$ Inelastic neutron scattering (INS) measurements show the formation of a two gap structure in $\mathrm{URu}_{2} \mathrm{Si}_{2}$ : one at the incommensurate wave vector $(1.4,0,0)$ and another at the commensurate wave vector $(1,0,0)$. The second gap is a good candidate for a signature of the $\mathrm{HO}$, since the commensurate peak disappears in the AF phase. ${ }^{4,7,11,12}$

The various theories that have been presented so far to explain the $\mathrm{HO}$ paradigm can be separated into sets of itinerant and localized models. Among the localized models, we can cite the multipolar models, ${ }^{13-17}$ and among the itinerant models, there are different conjectures, indicating that the HO-AF transition is either driven by spin-density wave, ${ }^{18-20}$ hybridization, ${ }^{21,22}$ orbital $\mathrm{AF},{ }^{23}$ or spin nematic order effects. ${ }^{24}$ The great advantage of our model is the ability to integrate in a natural way the $\mathrm{HO}$ and $\mathrm{AF}$ effects already in a realistic localized treatment. Strong arguments in favor of our proposal can be taken from the following experimental observations: the Kondo-like hybridization of the $5 f$ electrons revealed at $T_{K} \approx 60 \mathrm{~K}$ does not discriminate between $\mathrm{HO}$ and $\mathrm{AF}$ phases. ${ }^{25-29}$ The INS measurements, ${ }^{7,12}$ which are insensitive to charge fluctuations, show a clear signature of the HO. From this perspective, we believe that the hybridization effect is not crucial for the HO-AF transition observed in $\mathrm{URu}_{2} \mathrm{Si}_{2}$. Therefore, we neglect the charge fluctuations to begin with and we concentrate solely on the magnetic degrees of freedom.

The modulated spin liquid (MSL) in a two-dimensional (2D) square lattice was developed in our previous work ${ }^{30}$ in order to explain the hidden-order phase in $\mathrm{URu}_{2} \mathrm{Si}_{2}$. The $2 \mathrm{D}$ version of the MSL model provides a simple scenario for the $\mathrm{URu}_{2} \mathrm{Si}_{2}$, with the $\mathrm{HO}$ phase resulting from a quantum phase transition where the local magnetic moments of the AF phase melt, restoring the time-reversal symmetry and preserving the lattice-breaking symmetry. ${ }^{31}$ One general concept introduced by the MSL model is that the modulation of the SL reflects the structure of the magnetically ordered phase, as shown in Fig. 1(c). The transport measurements indicate a continuous transition from the HO to AF phase. The MSL is in this way the resonant-valence-bond relative of the AF phase.

In the present work, we develop a 3D MSL model in a realistic body-centered tetragonal lattice (BCT lattice). Our spin liquid (SL) framework in this $3 \mathrm{D}$ system is shown to be both realistic and experimentally motivated to explain the onset of the $\mathrm{HO}$ phase in $\mathrm{URu}_{2} \mathrm{Si}_{2}$. We propose a microscopic model to explain the HO-AF transition, where the magnetic interaction varies linearly with the lattice spacing.

The BCT lattice structure of $\mathrm{URu}_{2} \mathrm{Si}_{2}$ is depicted in Fig. 1 . For convenience, we use here the tetragonal basis $(\mathbf{a}, \mathbf{b}, \mathbf{c})$, which contains two $\mathrm{U}$ atoms per unit cell of the tetragonal lattice (T lattice). Experimentally, the lattice parameters are $|\mathbf{a}|=|\mathbf{b}|=4.124 \AA$ and $|\mathbf{c}|=9.5817 \AA$ in the HO phase, with less than $1 \%$ variation when the system is warmed up to room temperature. ${ }^{1}$

We start with a Heisenberg model Hamiltonian using the standard fermionic representation of quantum spins $1 / 2$,

$$
H_{0}=\sum_{\left\langle\mathbf{R}, \mathbf{R}^{\prime}\right\rangle, \sigma \sigma^{\prime}} J_{\mathbf{R} \mathbf{R}^{\prime}} \chi_{\mathbf{R} \sigma}^{\dagger} \chi_{\mathbf{R} \sigma^{\prime}} \chi_{\mathbf{R}^{\prime} \sigma^{\prime}}^{\dagger} \chi_{\mathbf{R}^{\prime} \sigma},
$$

where the fermion annihilation (creation) operators $\chi_{\mathbf{R} \sigma}^{(\dagger)}$ satisfy the local constraints $\sum_{\sigma=\uparrow, \downarrow} \chi_{\mathbf{R} \sigma}^{\dagger} \chi_{\mathbf{R} \sigma}=1$. For simplicity, 


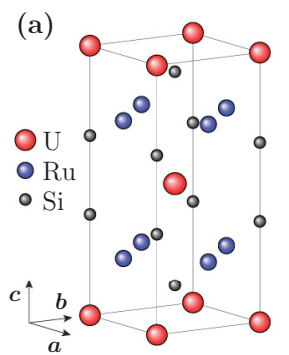

(b)

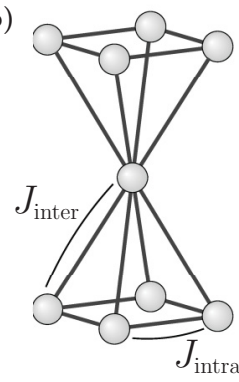

(c)
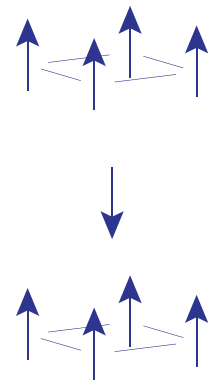

FIG. 1. (Color online) (a) The crystal structure of $\mathrm{URu}_{2} \mathrm{Si}_{2}$. The magnetism emerges from the $5 f$ electrons of $U$ atoms. (b) The BCT-lattice Bravais structure of the uranium atoms. The intralayer magnetic coupling is ferromagnetic, $J_{\text {intra }}<0$. The interlayer coupling is antiferromagnetic, $J_{\text {inter }}>0$. (c) The structure of the magnetic long-range order characterizing $\mathrm{URu}_{2} \mathrm{Si}_{2}$.

we consider only the magnetic interaction between nearestneighbor sites $\mathbf{R}$ and $\mathbf{R}^{\prime}$, as depicted in Fig. 1(b). We assume this nearest-neighbor interaction to be ferromagnetic $\left(J_{\mathbf{R R}^{\prime}}=J_{\text {intra }}<0\right)$ inside each layer and antiferromagnetic $\left(J_{\mathbf{R}^{\prime}}=J_{\text {inter }}>0\right)$ in between adjacent layers. This is the simplest and most natural interaction which can reproduce the magnetically ordered phase obtained experimentally at high pressure: ${ }^{5}$ an intralayer ferromagnetism together with interlayer antiferromagnetism [see Fig. 1(c)].

Generalizing the procedure of Ref. 30, the Heisenberg Hamiltonian (1) is decoupled for each bond $\mathbf{R} \mathbf{R}^{\prime}$ using appropriated Hubbard-Stratonovich transformations. We find the following Lagrangian:

$$
\begin{aligned}
\mathcal{L}_{0}= & \sum_{\mathbf{R} \sigma} \chi_{\mathbf{R} \sigma}^{\dagger}\left(\partial_{\tau}+\lambda_{\mathbf{R}}+\sigma \sum_{\mathbf{z}} m_{\mathbf{R}+\mathbf{z}}\right) \chi_{\mathbf{R} \sigma} \\
& -\sum_{\mathbf{R}} \lambda_{\mathbf{R}}+\sum_{n \sigma} \sum_{\left\langle\mathbf{R} \in L_{n}, \mathbf{R}^{\prime} \in L_{n \pm 1}\right\rangle}\left[\varphi_{\mathbf{R} \mathbf{R}^{\prime}} \chi_{\mathbf{R} \sigma}^{\dagger} \chi_{\mathbf{R}^{\prime} \sigma}+\text { c.c. }\right] \\
& +\sum_{n}\left(\sum_{\left\langle\mathbf{R} \in L_{n}, \mathbf{R}^{\prime} \in L_{n \pm 1}\right\rangle} \frac{2\left|\varphi_{\mathbf{R} \mathbf{R}^{\prime}}\right|^{2}}{J_{\text {inter }}}-\sum_{\left\langle\mathbf{R}, \mathbf{R}^{\prime}\right\rangle \in L_{n}} \frac{m_{\mathbf{R}} m_{\mathbf{R}^{\prime}}}{2 J_{\text {intra }}}\right),
\end{aligned}
$$

where $\lambda_{\mathbf{R}}$ represents the Lagrange multiplier introduced to satisfy the constraint of one particle per site, $L_{n}$ denotes the layer $n$, and the sum over $\mathbf{z}$ refers to the nearest neighbors within the same layer. In the following, the Hubbard-Stratonovich fields will be replaced by their constant, self-consistent, meanfield expressions, $\varphi_{\mathbf{R}^{\prime}}=-J_{\text {inter }} \sum_{\sigma}\left\langle\chi_{\mathbf{R}_{\sigma}}^{\dagger} \chi_{\mathbf{R}^{\prime} \sigma}\right\rangle$ and $m_{\mathbf{R}}=$ $J_{\text {intra }} \sum_{\sigma} \sigma\left\langle\chi_{\mathbf{R} \sigma}^{\dagger} \chi_{\mathbf{R} \sigma}\right\rangle$.

Note that this magnetic, intralayer-only, decoupling channel leads to a degenerate mean-field system, with each layer becoming effectively ferromagnetic, but with an easy axis completely decoupled from the other layers. This degeneracy does not distinguish an artificially fully ferromagnetic order from the expected AF order depicted by Fig. 1(c). A more general decoupling scheme would consist of splitting arbitrarily the interlayer interaction, $J_{\text {inter }} \equiv J_{\mathrm{SL}}+J_{\mathrm{AF}}$, following closely the procedure used in Ref. 30: the terms with $J_{\mathrm{AF}}$ and $J_{\mathrm{SL}}$ are decoupled in the magnetic and SL channels, respectively. At the mean-field level, the degeneracy is lifted by the contribution from the interlayer part of the local Weiss fields, i.e., the contribution originating from $J_{\mathrm{AF}}$ terms. Despite an apparent higher complexity, this generalized mean-field problem is formally identical to the one described originally by the Lagrangian (2). Indeed, considering the BCT lattice coordination numbers, this general decoupling scheme can be derived at the mean-field level from the one used here by simply mapping $J_{\text {intra }} \mapsto J_{\text {intra }}+2 J_{\mathrm{AF}}$ and $J_{\text {inter }} \mapsto J_{\mathrm{SL}}=$ $J_{\mathrm{inter}}-J_{\mathrm{AF}}$. As we will see next, what is remarkable here, with the BCT lattice, is that the competition between the magnetic and MSL orders is simply tunable by changing the ratio $\left(J_{\text {intra }}+2 J_{\mathrm{AF}}\right) /\left(J_{\text {inter }}-J_{\mathrm{AF}}\right)$, which is qualitatively independent of the arbitrary splitting if we take $J_{\text {inter }}=$ $J_{\mathrm{SL}}+J_{\mathrm{AF}}$. Therefore, in this work, we just assume $J_{\mathrm{AF}}=0$ and we consider $J_{\text {intra }} / J_{\text {inter }}$ as the new tuning parameter which is phenomenologically associated with pressure variations.

Experimentally, pressure has a direct effect on the ratio between the interlayer magnetic coupling $J_{\text {inter }}$ and the intralayer one $J_{\text {intra }}$. This is not standard for heavy-fermion systems, where pressure variations may often change the local-energy level of the $f$ electrons. This different phenomenological approach is supported here by strong experimental evidence: in $\mathrm{URu}_{2} \mathrm{Si}_{2}$, pressure favors a magnetic phase. Here, of course, the mechanism is not Doniach-like.

We introduce the Fourier transform of the fields,

$$
\begin{aligned}
\chi_{\mathbf{k} \sigma} & \equiv \frac{1}{\sqrt{N}} \sum_{\mathbf{R}} e^{-i \mathbf{k} \cdot \mathbf{R}} \chi_{\mathbf{R} \sigma}, \quad m_{\mathbf{k}} \equiv \frac{1}{\sqrt{N}} \sum_{\mathbf{R}} e^{-i \mathbf{k} \cdot \mathbf{R}} m_{\mathbf{R}}, \\
\varphi_{\mathbf{q}} & \equiv \frac{e^{i \theta_{\mathbf{q}}}}{2 \sqrt{N}} \sum_{n} \sum_{\left\langle\mathbf{R} \in L_{n}, \mathbf{R}^{\prime} \in L_{n+1}\right\rangle} e^{-i \mathbf{q} \cdot\left(\frac{\mathbf{R}+\mathbf{R}^{\prime}}{2}\right)} \varphi_{\mathbf{R} \mathbf{R}^{\prime}},
\end{aligned}
$$

where $N$ is the number of lattice sites. The phase factor $\theta_{\mathbf{q}} \equiv$ $\mathbf{q} \cdot \mathbf{R}_{0}$ is introduced in order to fix the origin of the bond lattice at real-space position $\mathbf{R}_{0} \equiv(\mathbf{a}+\mathbf{b}+\mathbf{c}) / 4$.

Hereafter, we will concentrate our analysis on the following mean-field parameters: the uniform SL $\Phi_{0} \equiv \varphi_{(0,0,0)} / \sqrt{N}$, the modulated SL $\Phi_{\mathbf{Q}} \equiv \varphi_{\mathbf{Q}} / \sqrt{N}$, and the Néel staggered magnetization $\mathrm{AF} S_{\mathbf{Q}_{\mathrm{AF}}} \equiv m_{\mathbf{Q}_{\mathrm{AF}}} / \sqrt{N}$. There is an important difference from the square-lattice MSL. ${ }^{30}$ Here, the equivalences between wave vectors $\mathbf{Q}$ for the $\varphi$ fields do not refer to the same Brillouin zones as the ones between $\mathbf{Q}_{\mathrm{AF}}$ for the $m$ fields. This is due to the fact that the magnetization fields are defined on the sites, while the SL fields are defined on the bonds. The symmetry group of the AF phase corresponds to a $\mathrm{T}$ lattice, and $\mathbf{Q}_{\mathrm{AF}}$ is thus defined modulo the first Brillouin zone of the T lattice. We consider that the MSL states, similarly to what happens in the AF phase, satisfy the T lattice translational symmetries, although different $\mathbf{Q}$ modulation vectors are defined modulo a larger Brillouin zone, characterizing the long-range order with $\mathrm{T}$ lattice periodicity but with different intrashell symmetry breaking (see Fig. 2).

For simplicity, we consider at most the case of one kind of modulation at a time in the presence of an homogeneous solution. If $\mathbf{Q}$ is the wave vector associated with the MSL, then $\mathbf{Q}_{\mathrm{AF}}$ is the wave vector associated with the AF. We get $m_{\mathbf{R}}=S_{\mathbf{Q}_{A F}} e^{i \mathbf{Q}_{A F} \cdot \mathbf{R}}, \varphi_{\mathbf{q}}=\Phi_{0} \sqrt{N} \delta_{\mathbf{q}}+\Phi_{\mathbf{Q}} \sqrt{N} \delta_{\mathbf{q}+\mathbf{Q}}$, where $\delta$ denotes the Kronecker $\delta$. Note that a completely equivalent ansatz can also be made in the direct bond lattice, similarly to 
(a)

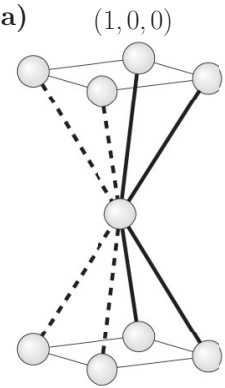

(b)

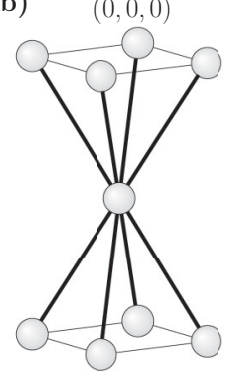

$(0,0,1)$

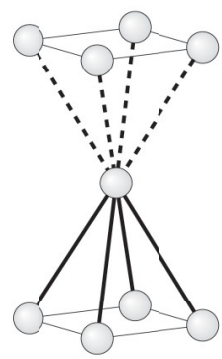

$(1,1,0)$

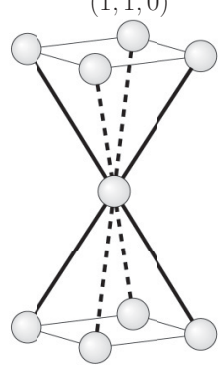

$(1,1,1)$
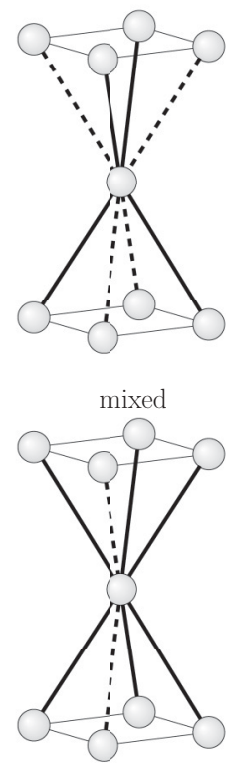

FIG. 2. Possible SL long-range ordered modulations represented on the unit cell of the T lattice. When it is well defined, the associated wave vector is indicated in reduced coordinates $(h, k, l)$. Dotted lines represent $\varphi_{\mathbf{R R}^{\prime}}=\varphi_{0}+\varphi_{\mathbf{Q}}$ and solid lines represent $\varphi_{\mathbf{R}^{\prime}}=\varphi_{0}-\varphi_{\mathbf{Q}}$ bond modulation. (a) The three different relevant odd MSL wave vectors considered. (b) Respectively, the homogeneous SL, an even wave vector that we exclude (see text), and a mixed modulation which may invoke more than one wave vector (not considered in this work).

what was done earlier in Ref. 30, namely, $\varphi_{\mathbf{R} \mathbf{R}^{\prime}}=\delta_{\mathbf{R}, \mathbf{R}^{\prime}+\mathbf{z}}\left(\varphi_{0}+\right.$ $\varphi_{\mathbf{Q}} e^{\left.-i \mathbf{Q} \cdot\left(\mathbf{R}+\mathbf{R}^{\prime}\right) / 2-i \theta_{\mathbf{Q}}\right)}$.

We introduce the following reduced notation for the SL modulation wave vectors: $\mathbf{Q} \equiv(h, k, l)$. The parity of the modulation can be obtained from the phase factor $e^{i \pi(h, k, l)}=$ \pm 1 . The sign $+(-)$ characterizes wave vectors with even (odd) parity. The only possible MSLs characterized by a single modulation wave vector have odd parity. The definition of parity can be extended to the AF wave vector. We find here that the parity of $\mathbf{Q}_{\mathrm{AF}}$ is odd [see Fig. 1(c)]. For simplification, we consider here MSL wave vectors with either one or three modulations. Due to the $\mathbf{a} \rightarrow \mathbf{b}$ symmetry, we finally need to compare only three types of modulations (see Fig. 2). All of these wave vectors break BCT lattice symmetry and have the periodicity of a $\mathrm{T}$ lattice: $\mathbf{Q}_{1} \equiv(1,0,0)$ breaks the $C_{4}$ rotational symmetry, characterizing an orthorhombic lattice, and is compatible with the state discussed in Refs. 24 and 29, $\mathbf{Q}_{2} \equiv(0,0,1)$ may break a mirror symmetry, and $\mathbf{Q}_{3} \equiv(1,1,1)$ clearly belongs to the $\mathrm{T}$ lattice group.

The selection between different modulation vectors $\mathbf{Q}$ is obtained by comparing the corresponding minimized free energies per site, which is given by

$$
\begin{aligned}
F\left(\lambda_{0}, \Phi_{0}, \Phi_{\mathbf{Q}}, S_{\mathbf{Q}_{\mathrm{AF}}}\right)= & -\frac{k_{B} T}{N} \sum_{\mathbf{k}} \sum_{\alpha= \pm} \ln \left[1+e^{-\beta \Omega_{\mathbf{k}}^{\alpha}}\right] \\
& -\lambda_{\mathbf{0}}+\frac{4}{J_{\text {inter }}}\left[\left|\Phi_{0}\right|^{2}+\left|\Phi_{\mathbf{Q}}\right|^{2}\right] \\
& -\frac{2}{J_{\text {intra }}}\left|S_{\mathbf{Q}_{\mathrm{AF}}}\right|^{2},
\end{aligned}
$$

(a)

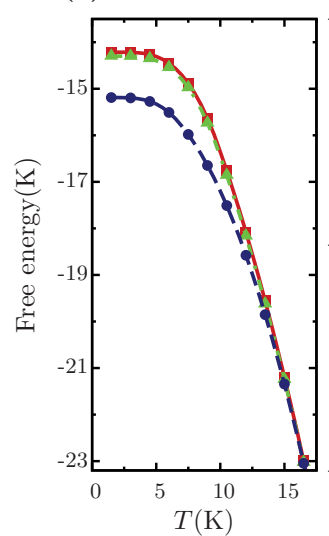

(b)

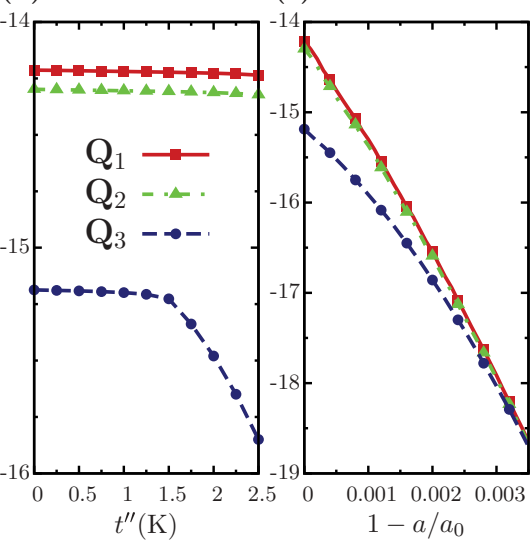

FIG. 3. (Color online) Free energy of three modulating vectors $\mathbf{Q}_{1}=(1,0,0), \mathbf{Q}_{2}=(0,0,1)$, and $\mathbf{Q}_{3}=(1,1,1)$ as a function of (a) $T$ for $t^{\prime \prime}=0 \mathrm{~K}$ and $a=a_{0}$, (b) hopping for $T=2 \mathrm{~K}$ and $a=a_{0}$, and (c) $1-a / a_{0}$ for $T=2 \mathrm{~K}$ and $t^{\prime \prime}=0 \mathrm{~K}$. The parameters used were $J_{\text {intra }}\left(a_{0}\right)=-6.5 \mathrm{~K}, J_{\text {inter }}\left(a_{0}\right)=37 \mathrm{~K}$, and $\mathcal{B}=800 \mathrm{~K}$, as described in the text.

where the sum over $\mathbf{k}$ is taken over the full Brillouin zone and the eigenenergies are given by

$$
\Omega_{\mathbf{k}}^{ \pm}=\lambda_{0} \pm 4 \sqrt{S_{\mathbf{Q}_{\mathrm{AF}}}^{2}+4 \Phi_{0}^{2} \gamma_{1, \mathbf{k}}^{2}+4\left|\Phi_{\mathbf{Q}}\right|^{2} \gamma_{2, \mathbf{k}, \mathbf{Q}}^{2}},
$$

with $\quad \gamma_{1, \mathbf{k}}=\cos \left(\frac{k_{a}}{2}\right) \cos \left(\frac{k_{b}}{2}\right) \cos \left(\frac{k_{c}}{2}\right) \quad$ and $\quad \gamma_{2, \mathbf{k}, \mathbf{Q}}=$ $\cos \left(\frac{k_{a}}{2}+\frac{Q_{a}}{4}\right) \cos \left(\frac{k_{b}}{2}+\frac{Q_{b}}{4}\right) \cos \left(\frac{k_{c}}{2}+\frac{Q_{c}}{4}\right)$. For a given modulating wave vector $\mathbf{Q}$, the staggered magnetization $S_{\mathbf{Q}_{\mathrm{AF}}}$ and the homogeneous and modulated SL parameters $\Phi_{0}$ and $\Phi_{\mathbf{Q}}$ are obtained directly from the minimization of the free-energy function. The free energy is calculated minimizing Eq. (3), using Powell's method, ${ }^{32}$ with the auxiliary equation to fix the number of $n_{f}, 1=\frac{1}{N} \sum_{\mathbf{k}} \sum_{\alpha= \pm} \frac{1}{1+e^{\beta \Omega_{\mathbf{k}}^{\alpha}}}$.

Figure 3 depicts the behavior of the free energy for the three different wave vectors $\mathbf{Q}_{1}, \mathbf{Q}_{2}$ and $\mathbf{Q}_{3}$. We find, by varying the different physical parameters of the model, that the minimum is always obtained for the wave vector $\mathbf{Q}_{3}=(1,1,1)$. These states, which correspond to the space group $134 \mathrm{P}_{2} / \mathrm{nnm}$ also appear to be compatible with the crystallographic analysis of $\mathrm{URu}_{2} \mathrm{Si}_{2}$ made by Harima et al. ${ }^{15}$

A next-nearest-neighbor hopping $t^{\prime \prime}$ may be included in order to phenomenologically take into account some frustration and intraplane spin liquid contribution. In this case, the intralayer term is the same for momenta $\mathbf{k}$ and $\mathbf{k}+\mathbf{Q}$, and the extension of our model is directly obtained if we perform the change $\Omega_{\mathbf{k}}^{ \pm} \mapsto \Omega_{\mathbf{k}}^{ \pm}+t^{\prime \prime} \Phi_{0} \cos \left(k_{a}\right) \cos \left(k_{b}\right)$.

Motivated by the pressure experiments which are specially dedicated to the anisotropy and the uniaxial effects, ${ }^{8-10}$ we relate our microscopic interaction with pressure. Bourdarot et $a l .{ }^{8}$ showed that the uniaxial stress is the relevant variational parameter to change the behavior of the system. They considered that the deformation is in the linear elastic regime. In this work, we propose that our parameters $J_{\text {intra }}$ and $J_{\text {inter }}$ also vary linearly with the lattice parameter $a$. The variation can be simply written as $J_{\text {inter }}(a)=J_{\text {inter }}\left(a_{0}\right)+\mathcal{B}_{1}\left(1-a / a_{0}\right)$ and $J_{\text {intra }}(a)=J_{\text {intra }}\left(a_{0}\right)-\mathcal{B}_{2}\left(1-a / a_{0}\right)$, where $a_{0}$ is the value of the lattice parameter in ambient pressure. 


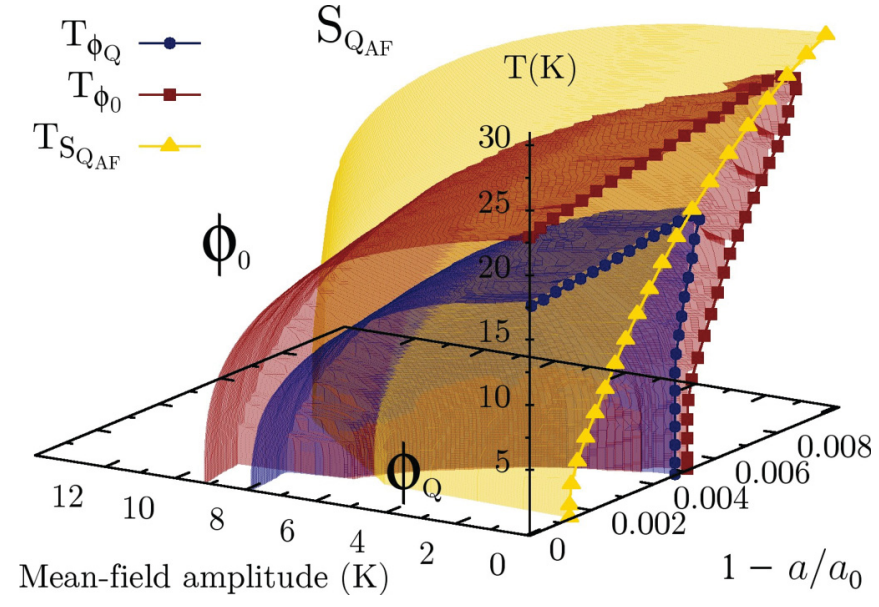

FIG. 4. (Color online) The phase diagram for the modulation $\mathbf{Q}_{3}$ as a function of deformation $\left(1-a / a_{0}\right)$ and $T$. Inside the surfaces, the mean-field amplitudes are different from zero. There is a region where the three order parameters coexist. The circle (blue), square (red), and triangle (yellow) lines represent the critical temperatures for the parameters $\Phi_{\mathbf{Q}}, \Phi_{0}$, and $S_{\mathbf{Q}_{\mathrm{AF}}}$, respectively. The parameters used are $t^{\prime \prime}=2.5 \mathrm{~K}, J_{\text {intra }}\left(a_{0}\right)=-6.5 \mathrm{~K}, J_{\text {inter }}\left(a_{0}\right)=37 \mathrm{~K}$, and $\mathcal{B}=800 \mathrm{~K}$.

Here, the fitting parameters chosen to produce a phase diagram in qualitatively good agreement with experiment are as follows: $J_{\text {inter }}\left(a_{0}\right)=37 \mathrm{~K}$ is chosen to obtain $T_{0}=17.5 \mathrm{~K}$ and $J_{\text {intra }}\left(a_{0}\right)=-6.5 \mathrm{~K}$ is chosen to obtain the best approximated value for the critical stress $1-a / a_{0} \approx 1.45 \times 10^{-3}$. To have good agreement with experiment, we also choose the linear coefficient of $J_{\text {inter }}(a), \mathcal{B}_{1}$ to have the same slope of $T_{0}$, as observed experimentally, and we define $\mathcal{B}_{2}=\mathcal{B}_{1} \equiv \mathcal{B}$ for simplicity. Both $J_{\text {inter }}(a)$ and $J_{\text {intra }}(a)$ increase their absolute values when $1-a / a_{0}$ increases. Our choice of the interaction parameters' variation is, of course, a simplified view of the experiment: if we apply an uniaxial stress, then the in-plane lattice parameters become different. In our case, both in-plane parameters decrease in the same way.

The resulting phase diagram is shown in Fig. 4. The variation on $1-a / a_{0}$ shows very good agreement with the experimental results. We define $T_{\Phi_{Q}}, T_{\Phi_{0}}$, and $T_{S_{Q_{A F}}}$ as the critical temperatures for the parameters $\Phi_{\mathbf{Q}}, \Phi_{0}$, and $S_{\mathbf{Q}_{A F}}$, respectively. Increasing $1-a / a_{0}$, the MSL critical temperature $T_{\Phi_{\mathrm{Q}}}$ increases linearly until it reaches the $\mathrm{AF}$ ordering temperature $T_{\mathrm{Q}_{\mathrm{AF}}}$ and then it goes to zero, showing a reentrance behavior due to the presence of the hopping $t^{\prime \prime}$. The homogeneous component $T_{\Phi_{0}}$ shows a similar variation, although with a bigger amplitude for $t^{\prime \prime}$ different from zero. In our model, $\Phi_{0}$ persists for big values of $T$ or $1-a / a_{0}$, but with a small intensity. For the sake of simplicity, we define $T_{\Phi_{0}}$ when $\Phi_{0}=0.6 \mathrm{~K}$. The $T_{S_{\mathrm{Q}_{A F}}}$ also increases with $1-a / a_{0}$, but it shows two different behaviors: at first, a fast increase when it is inside the SL phase and a linear increase outside the SL. Here the effect of hopping is visible: without this effect, $T_{S_{\mathrm{Q}_{A F}}}$ will present just linear slopes. A first-order transition can also be obtained for $T_{S_{\mathrm{Q}_{\mathrm{AF}}}}$ inside the MSL phase when $t^{\prime \prime}$ is present.

The entropy per site $S \equiv-\partial F / \partial T$ and the specific-heat coefficient $C / T \equiv \partial S / \partial T$ as a function of temperature are plotted in Fig. 5. Here, we select two values of the relative

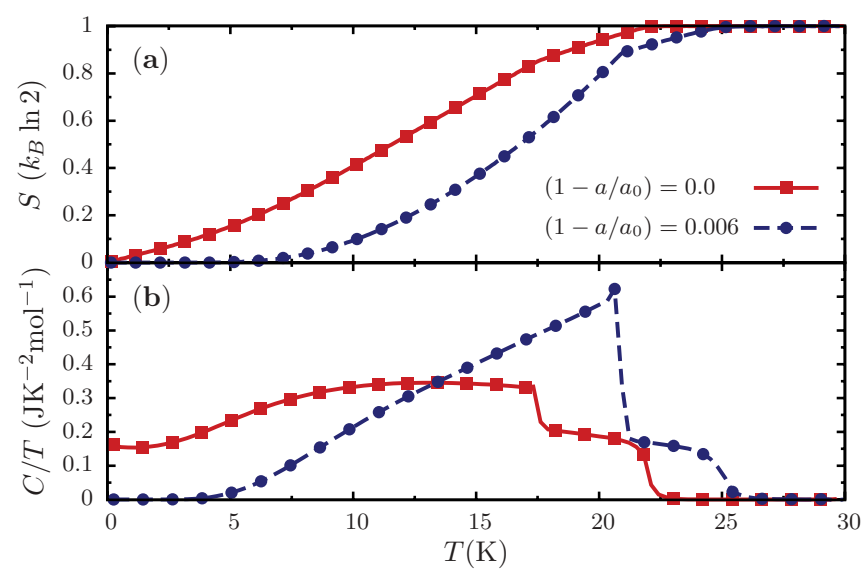

FIG. 5. (Color online) The (a) entropy per site and (b) specificheat coefficient as a function of temperature for $a=a_{0}$, i.e., the MSL phase, and for $\left(1-a / a_{0}\right)=0.006$, i.e., the AF phase. The values of the physical parameters and the interactions are taken from the self-consistent results for $\mathbf{Q}=\mathbf{Q}_{3}$ (see Fig. 4).

lattice parameter $a / a_{0}$ to describe the thermodynamics in the two different phases: the MSL phase is analyzed for $a=a_{0}$ and the AF phase is analyzed for $1-a / a_{0}=0.006$. Similar behaviors are observed in both phases, in agreement with experimental observations. The specific heat shows a peaklike discontinuity at the transition temperatures and the entropy freezing is of the same order, $k_{B} \ln 2$. Secondary features observed for $C / T$ at higher $T$ are due to the presence of a finite homogeneous spin liquid phase, $\Phi_{0}$.

In conclusion, we have developed a modulated spin liquid model in the realistic three-dimensional BCT lattice. This provides a simple scenario for $\mathrm{URu}_{2} \mathrm{Si}_{2}$, where the hidden order results from a quantum phase transition with a very unusual behavior: the magnetic moments of the AF phase melt at low pressure, restoring the time-reversal symmetry, but the lattice symmetry breaking is still present. We analyzed how this SL melting in a BCT lattice can lead to different modulation wave vectors, among which $\mathbf{Q}_{3}=(1,1,1)$ is found to be the most stable energetically. The theoretical phase diagram reproduces qualitatively well what is observed experimentally for $\mathrm{URu}_{2} \mathrm{Si}_{2}$. We identify a second-order transition at $T_{0} \approx 17.5 \mathrm{~K}$ and a first-order transition from the MSL phase to the AF phase at low temperature. A strong point of our model is that it naturally provides a stabilization of the AF phase with pressure. In this way, the scenario for the AF to $\mathrm{HO}$ melting is non-Doniach-like and does not require a Kondo effect, even if it is also present in the system. In addition, our assumption that both magnetic coupling $J_{\text {intra }}$ and $J_{\text {inter }}$ increase linearly with the deformation of lattice spacing describes very well the experimental phase diagram. ${ }^{8}$

Our results clearly show that the choice of an appropriate modulation vector is crucial for the stability of the MSL phase. This could be directly checked experimentally by INS measurements. By comparing all crystallographic directions, one could find clear evidence for what this preferable modulation might be. Raman-scattering and nuclear magnetic resonance (NMR) experiments could also provide other independent checks of our results since the orientation dependence of the Raman or NMR spectrum could establish whether or not 
the modulation is indeed characterized by our $\mathbf{Q}_{3}$ vector. We believe that our study is a very good test for a MSL paradigm.

We acknowledge the financial support of Capes-Cofecub $\mathrm{Ph}$ 743-12. This research was also supported in part by the Brazilian Ministry of Science, Technology and Innovation (MCTI) and the Conselho Nacional de Desenvolvimento Científico e Tecnológico (CNPq). Finally, we thank M. R.
Norman for his help and for innumerable discussions and F. Bourdarot for carefully reading an earlier version of this paper and for very useful comments. We also acknowledge C. Lacroix, P. Coleman, H. Harima and K. Miyake for discussions. Research was carried out with the aid of the High Performance Computer System SGI Altix UV 1000 of the IIP-UFRN, Natal, Brazil.
${ }^{1}$ T. T. M. Palstra, A. A. Menovsky, J. van den Berg, A. J. Dirkmaat, P. H. Kes, G. J. Nieuwenhuys, and J. A. Mydosh, Phys. Rev. Lett. 55, 2727 (1985).

${ }^{2}$ J. A. Mydosh and P. M. Oppeneer, Rev. Mod. Phys. 83, 1301 (2011).

${ }^{3}$ M. B. Maple, J. W. Chen, Y. Dalichaouch, T. Kohara, C. Rossel, M. S. Torikachvili, M. W. McElfresh, and J. D. Thompson, Phys. Rev. Lett. 56, 185 (1986).

${ }^{4}$ C. Broholm, J. K. Kjems, W. J. L. Buyers, P. Matthews, T. T. M. Palstra, A. A. Menovsky, and J. A. Mydosh, Phys. Rev. Lett. 58, 1467 (1987).

${ }^{5}$ H. Amitsuka, K. Matsuda, I. Kawasaki, K. Tenya, and M. Yokoyama, J. Magn. Magn. Mater. 310, 214 (2007).

${ }^{6}$ E. Hassinger, G. Knebel, K. Izawa, P. Lejay, B. Salce, and J. Flouquet, Phys. Rev. B 77, 115117 (2008).

${ }^{7}$ A. Villaume, F. Bourdarot, E. Hassinger, S. Raymond, V. Taufour, D. Aoki, and J. Flouquet, Phys. Rev. B 78, 012504 (2008).

${ }^{8}$ F. Bourdarot, N. Martin, S. Raymond, L. P. Regnault, D. Aoki, V. Taufour, and J. Flouquet, Phys. Rev. B 84, 184430 (2011).

${ }^{9}$ K. Bakker, A. Devisser, E. Bruck, A. A. Menovsky, and J. J. M. Franse, J. Magn. Magn. Mater. 108, 63 (1992).

${ }^{10}$ M. Yokoyama, H. Amitsuka, K. Tenya, K. Watanabe, S. Kawarazaki, H. Yoshizawa, and J. A. Mydosh, Phys. Rev. B 72, 214419 (2005).

${ }^{11}$ F. Bourdarot, B. Fak, K. Habicht, and K. Prokes, Phys. Rev. Lett. 90, 067203 (2003).

${ }^{12}$ C. R. Wiebe, J. A. Janik, G. J. MacDougall, G. M. Luke, J. D. Garrett, H. D. Zhou, Y. J. Jo, L. Balicas, Y. Qiu, J. R. D. Copley et al., Nature Phys. 3, 96 (2007).

${ }^{13}$ F. Cricchio, F. Bultmark, O. Granas, and L. Nordstrom, Phys. Rev. Lett. 103, 107202 (2009).

${ }^{14}$ K. Haule and G. Kotliar, Europhys. Lett. 89, 57006 (2010).

${ }^{15}$ H. Harima, K. Miyake, and J. Flouquet, J. Phys. Soc. Jpn. 79, 4 (2010).

${ }^{16}$ A. I. Toth and G. Kotliar, Phys. Rev. Lett. 107, 266405 (2011).
${ }^{17}$ H. Kusunose and H. Harima, J. Phys. Soc. Jpn. 80, 084702 (2011).

${ }^{18}$ H. Ikeda and Y. Ohashi, Phys. Rev. Lett. 81, 3723 (1998).

${ }^{19}$ V. P. Mineev and M. E. Zhitomirsky, Phys. Rev. B 72, 014432 (2005).

${ }^{20}$ S. Elgazzar, J. Rusz, M. Amft, P. M. Oppeneer, and J. A. Mydosh, Nature Mater. 8, 337 (2009).

${ }^{21}$ A. V. Balatsky, A. Chantis, H. P. Dahal, D. Parker, and J. X. Zhu, Phys. Rev. B 79, 214413 (2009).

${ }^{22}$ P. S. Riseborough, B. Coqblin, and S. G. Magalhaes, Phys. Rev. B 85, 165116 (2012).

${ }^{23}$ P. Chandra, P. Coleman, J. A. Mydosh, and V. Tripathi, Nature (London) 417, 831 (2002).

${ }^{24}$ S. Fujimoto, Phys. Rev. Lett. 106, 196407 (2011).

${ }^{25}$ F. Bourdarot, E. Hassinger, S. Raymond, D. Aoki, V. Taufour, L.-P. Regnault, and J. Flouquet, J. Phys. Soc. Jpn. 79, 064719 (2010).

${ }^{26}$ A. R. Schmidt, M. H. Hamidian, P. Wahl, F. Meier, A. V. Balatsky, J. D. Garrett, T. J. Williams, G. M. Luke, and J. C. Davis, Nature (London) 465, 570 (2010).

${ }^{27}$ P. Aynajian, E. H. da Silva Neto, C. V. Parker, Y. Huang, A. Pasupathy, J. Mydosh, and A. Yazdani, Proc. Natl. Acad. Sci. USA 107, 10383 (2010).

${ }^{28}$ X. Lu, F. Ronning, P. H. Tobash, K. Gofryk, E. D. Bauer, and J. D. Thompson, Phys. Rev. B 85, 020402 (2012).

${ }^{29}$ S. Tonegawa, K. Hashimoto, K. Ikada, Y.-H. Lin, H. Shishido, Y. Haga, T. D. Matsuda, E. Yamamoto, Y. Onuki, H. Ikeda et al., Phys. Rev. Lett. 109, 036401 (2012).

${ }^{30}$ C. Pépin, M. R. Norman, S. Burdin, and A. Ferraz, Phys. Rev. Lett. 106, 106601 (2011).

${ }^{31}$ R. Okazaki, T. Shibauchi, H. J. Shi, Y. Haga, T. D. Matsuda, E. Yamamoto, Y. Onuki, H. Ikeda, and Y. Matsuda, Science 331, 439 (2011).

${ }^{32}$ W. H. Press, S. A. Teukolsky, W. T. Vetterling, and B. P. Flannery, Numerical Recipes in Fortran 77: The Art of Scientific Computing (Cambridge University Press, New York, 1992). 